\title{
Inorganic and metal organic nanocomposites for cascade-responsive imaging and photochemical synergistic effects
}

Xiaojun Hu,,$^{\dagger}$ Zhikang Zhu, ${ }^{\dagger+}$ Haibin Dong,${ }^{\dagger}$ Xiaoyu Zhu, ${ }^{\dagger}$ Han Zhu, ${ }^{\dagger}$ Kazuma Ogawa,${ }^{\S}$

Akira Odani, ${ }^{\S}$ Kwangnak Koh," Hongxia Chen ${ }^{\dagger}$

$\dagger$ Center for Molecular Recognition and Biosensing, School of Life Sciences, Shanghai University, Shanghai 200444, PR China.

$\ddagger$ Shanghai Key Laboratory of Bio-Energy Crop, School of Life Sciences, Shanghai University, Shanghai 200444, PR China.

$\S$ College of Medical, Pharmaceutical and Health Sciences, Kanazawa University, Kanazawa 920-1192, Japan.

// Institute of General Education, Pusan National University, Busan 609-735, Republic of Korea.

*Corresponding authors.

E-mail address: hxchen@shu.edu.cn (H. Chen) 


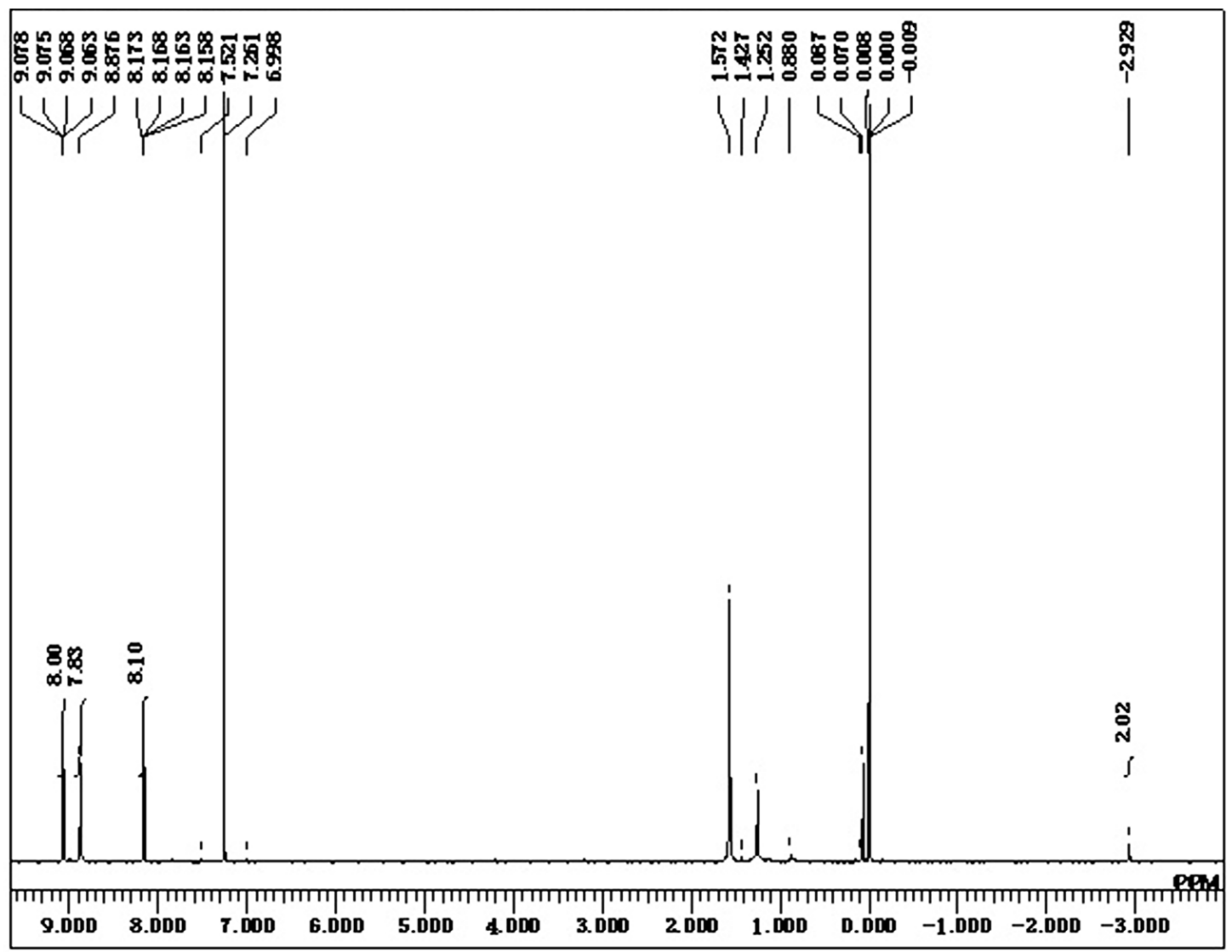

Figure. $\mathbf{S 1}{ }^{1} \mathrm{H}$ NMR spectrum of $\mathrm{H}_{2} \mathrm{TPyP}$ in $\mathrm{CDCl}_{3}$. 


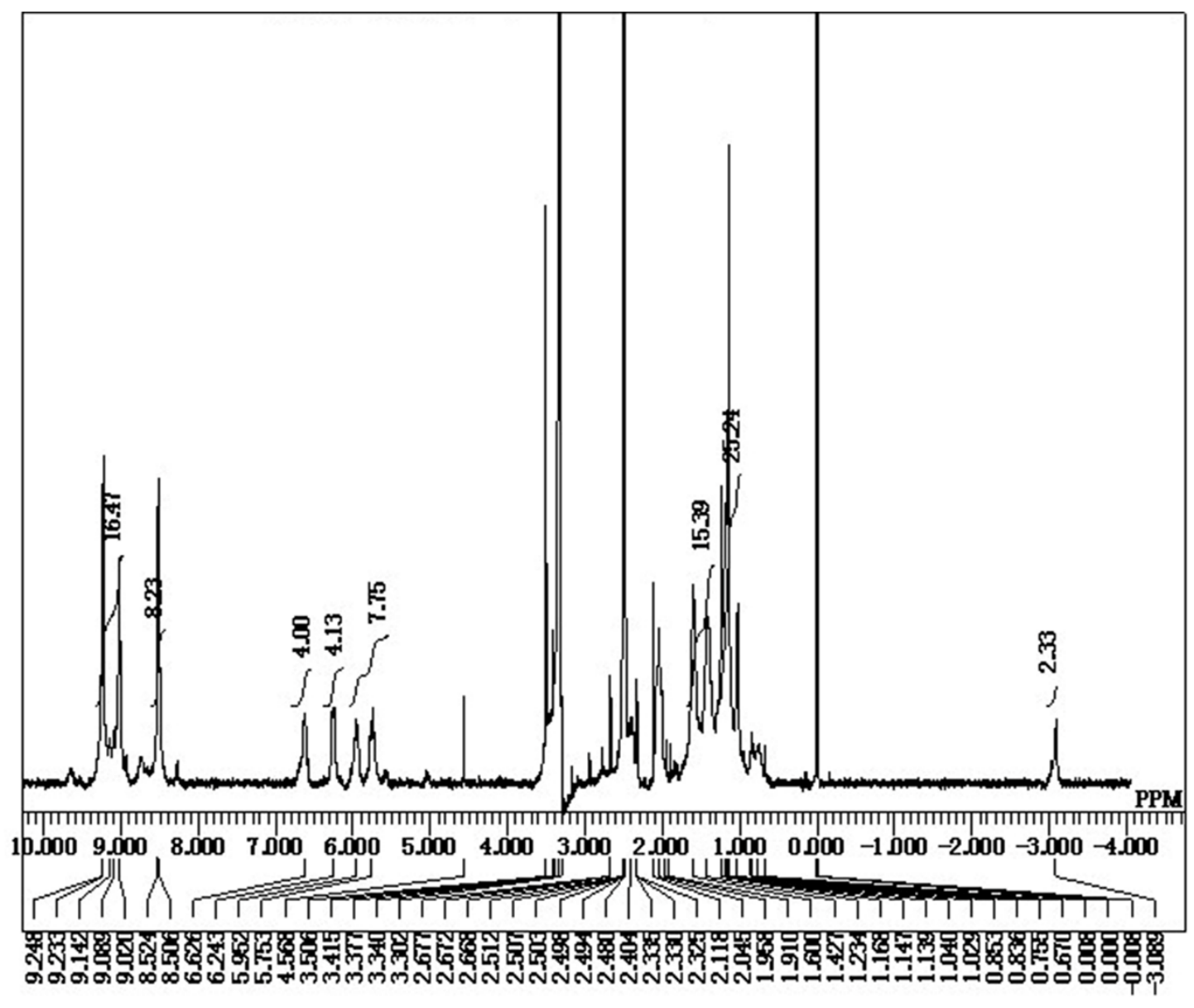

Figure. S2 ${ }^{1} \mathrm{H}$ NMR spectrum of $4 \mathrm{Pt}($ dach $)$ ClTPyP in DMSO- $d_{6}$. 


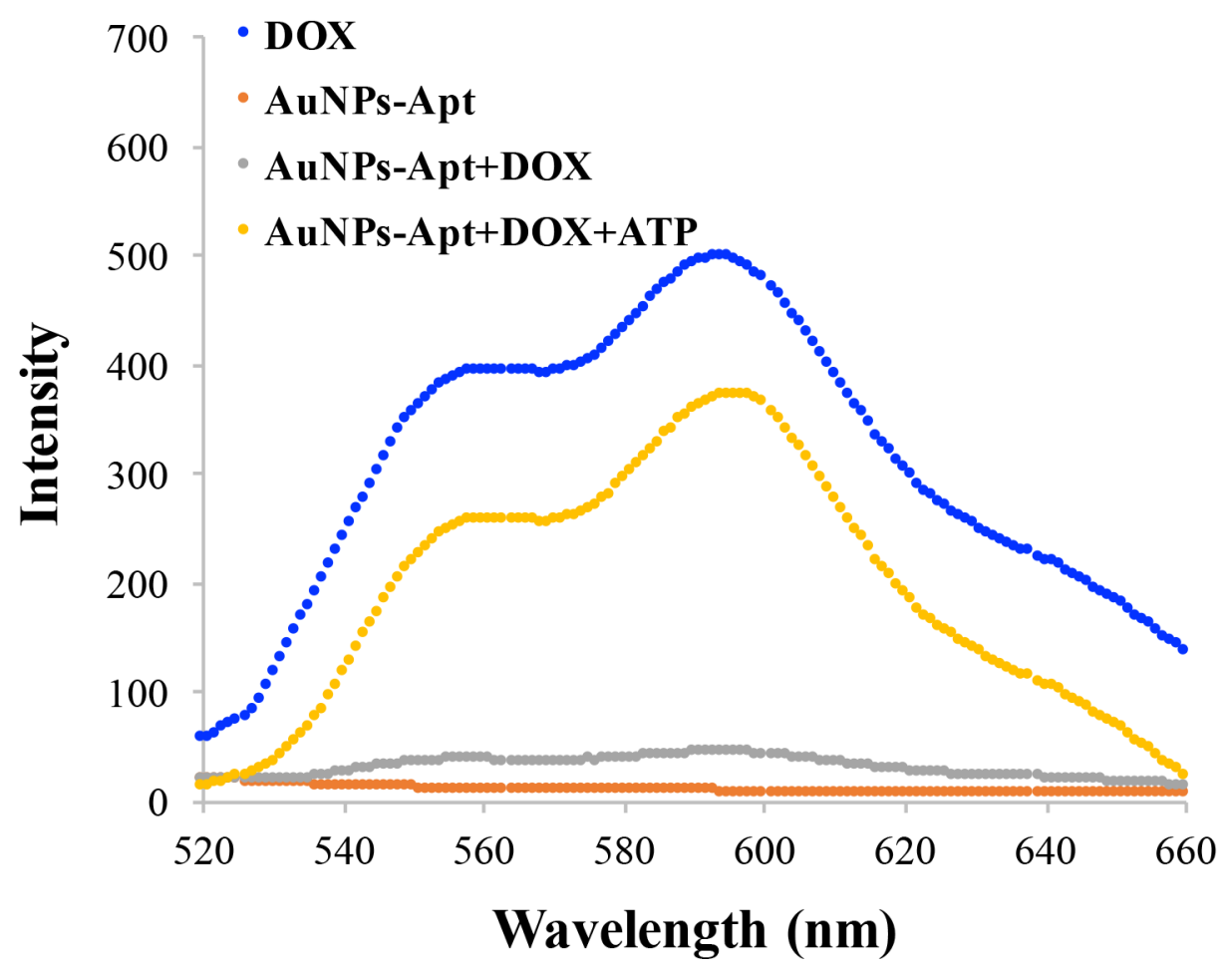

Figure. S3 The fluorescence spectra of DOX, DNA duplex-functional AuNPs, DOX inserted into the DNA duplex, and ATP effect on the DOX inserted AuNPs-Apt in pH 7.4 PBS buffer.

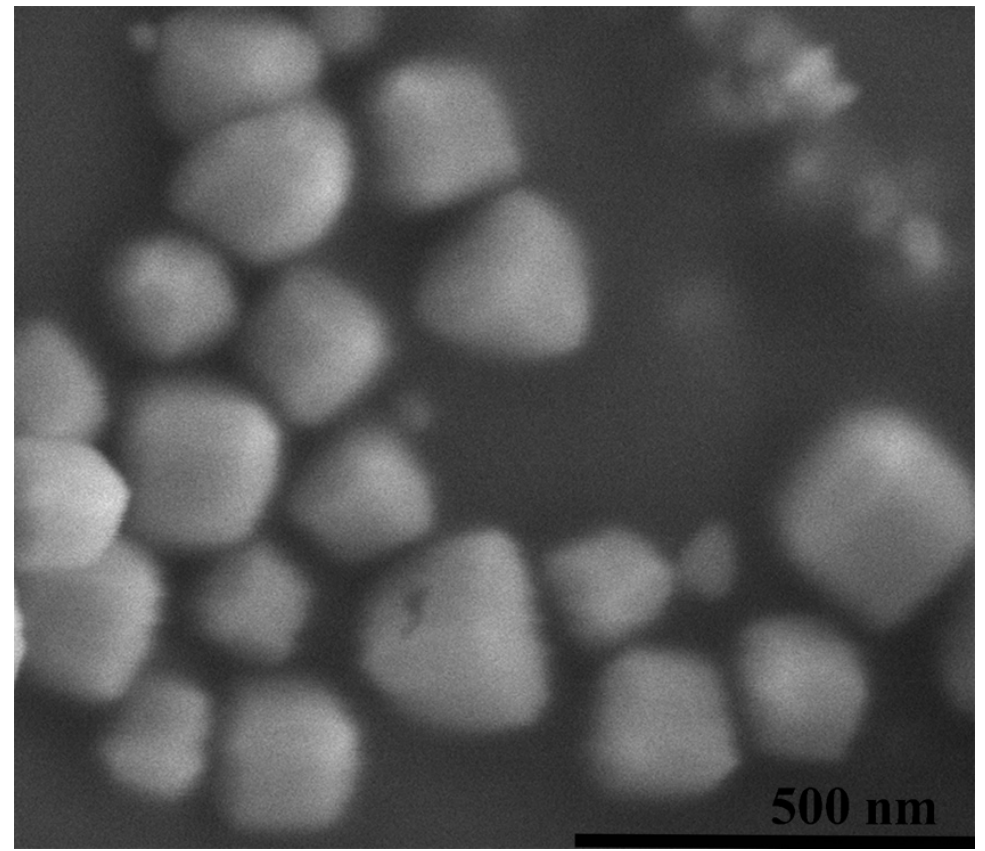

Figure. S4 SEM image of AuNPs-DOX/PtTPyP@ZIF-8. 


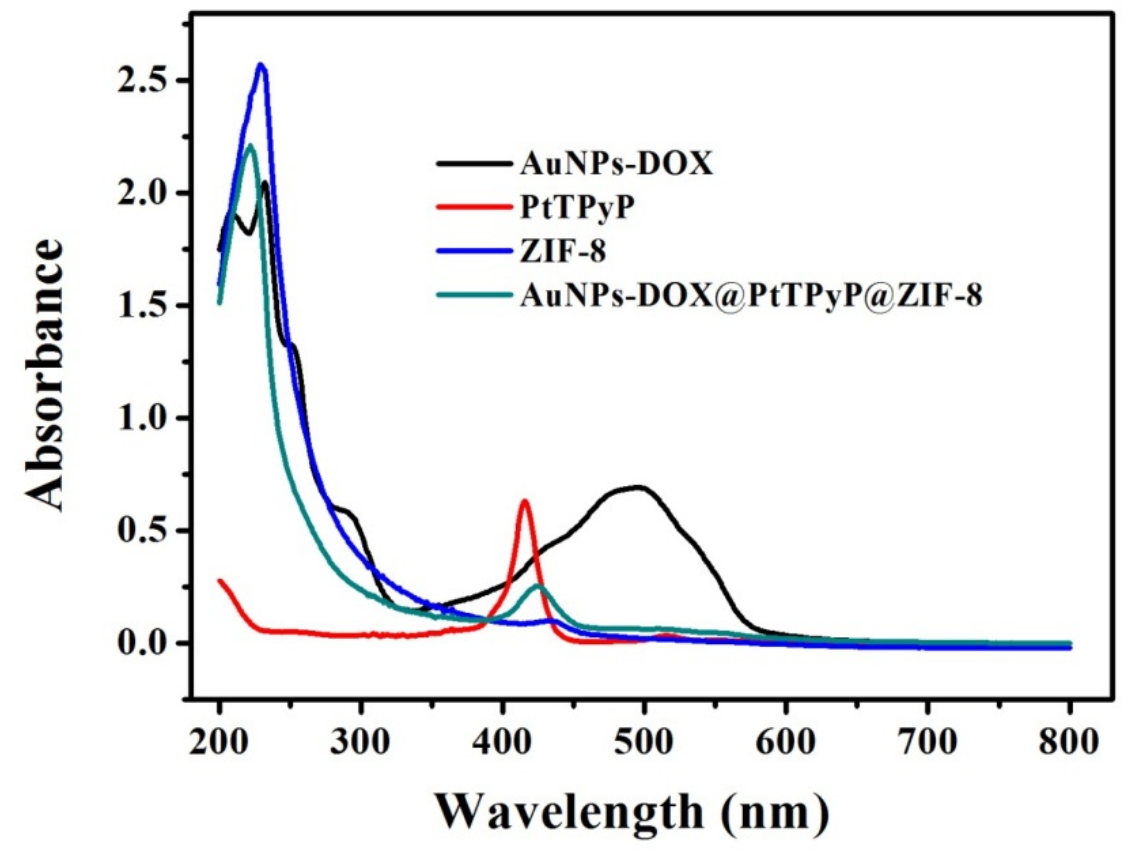

Figure. S5 UV-vis spectra of AuNPs-DOX, PtTPyP, ZIF-8 and AuNPs-DOX/PtTPyP@ZIF-8 in $\mathrm{H}_{2} \mathrm{O}$.

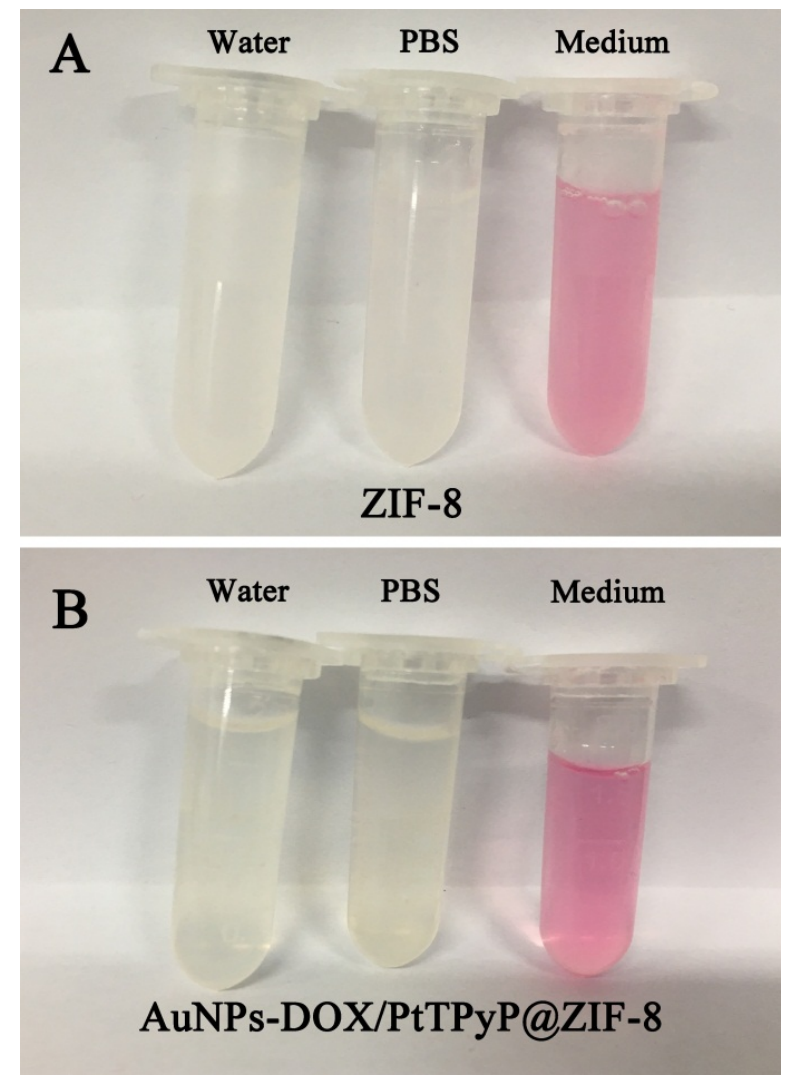

Figure. S6 Digital photos of ZIF-8 (A) and AuNPs-DOX/PtTPyP@ZIF-8 solution (B) after soaking in water, phosphate buffered saline and cell culture medium after $24 \mathrm{~h}$. 

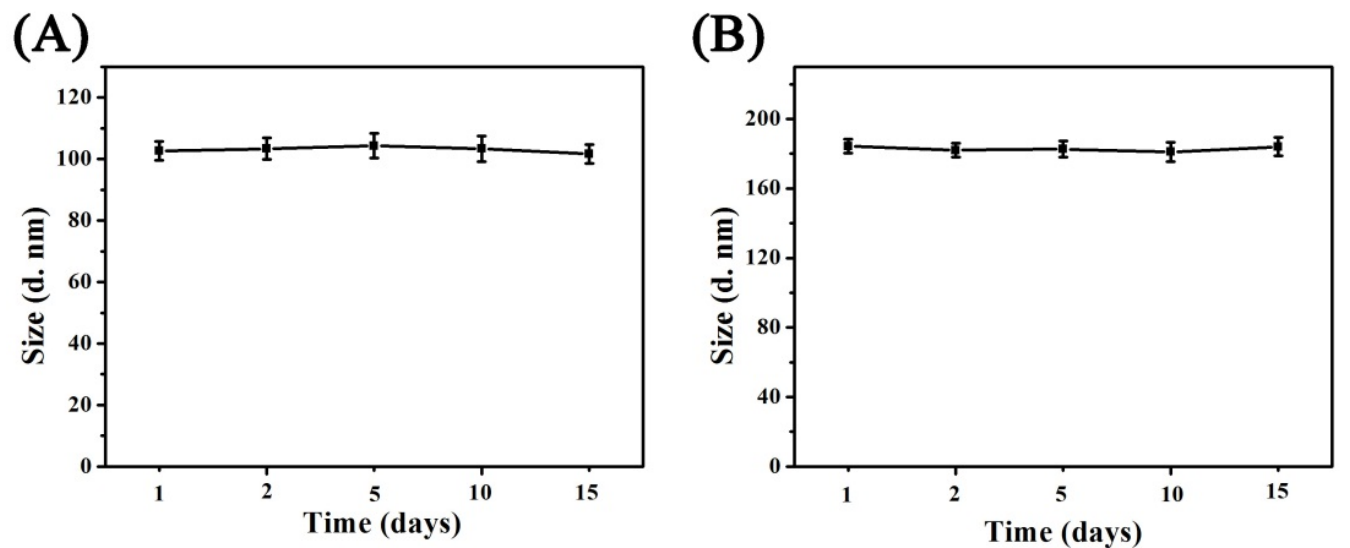

Figure. $\quad$ S7 DLS analysis of time-variation of ZIF-8 (A) and AuNPs-DOX/PtTPyP@ZIF-8 nanomaterials (B).

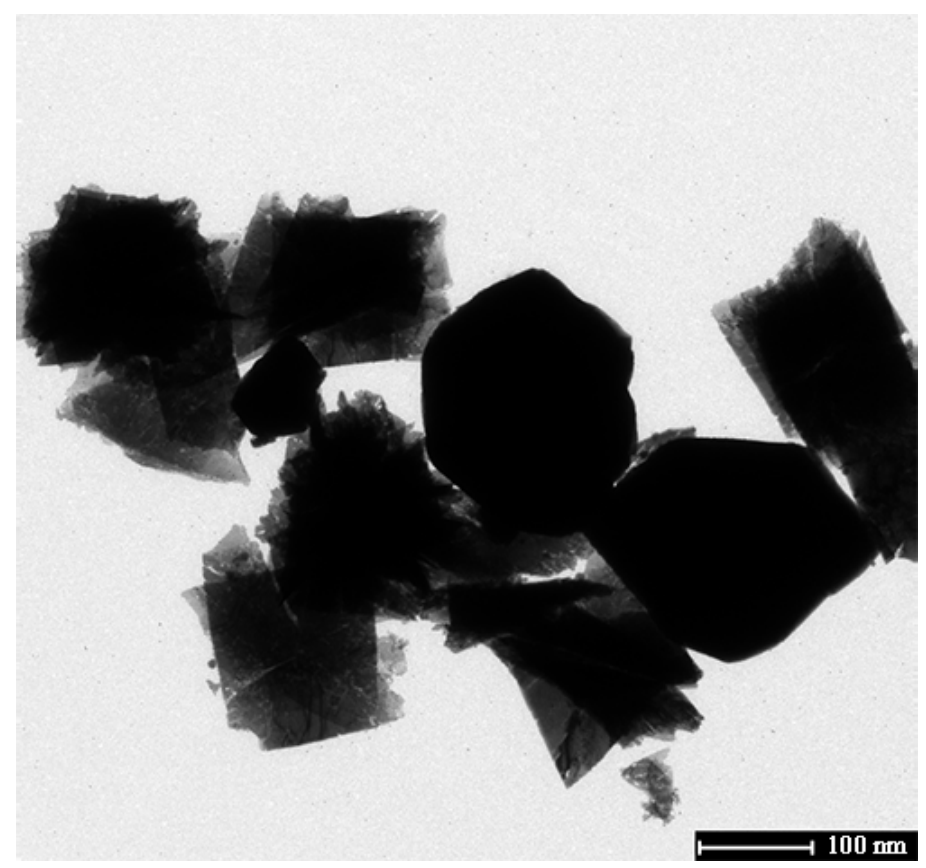

Figure. S8 TEM image of AuNPs-DOX/PtTPyP@ZIF-8 in PBS buffer solution at pH 5.0 . 

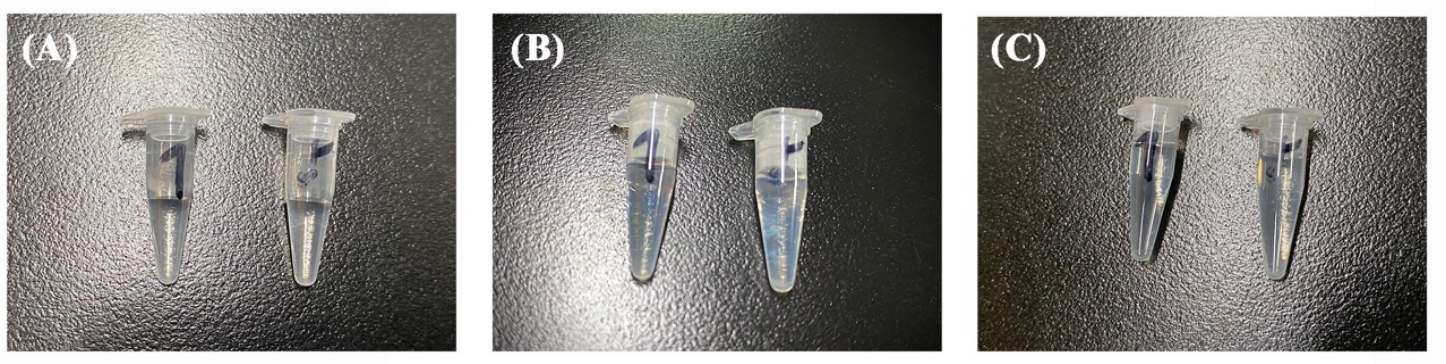

Figure. S9 The reaction of ZIF-8 with sodium hydroxide after ZIF-8 was treated by the solutions of $\mathrm{pH} 5.0$ and 7.4. (A) before reaction; (B) precipitation; (C) precipitation dissolution.
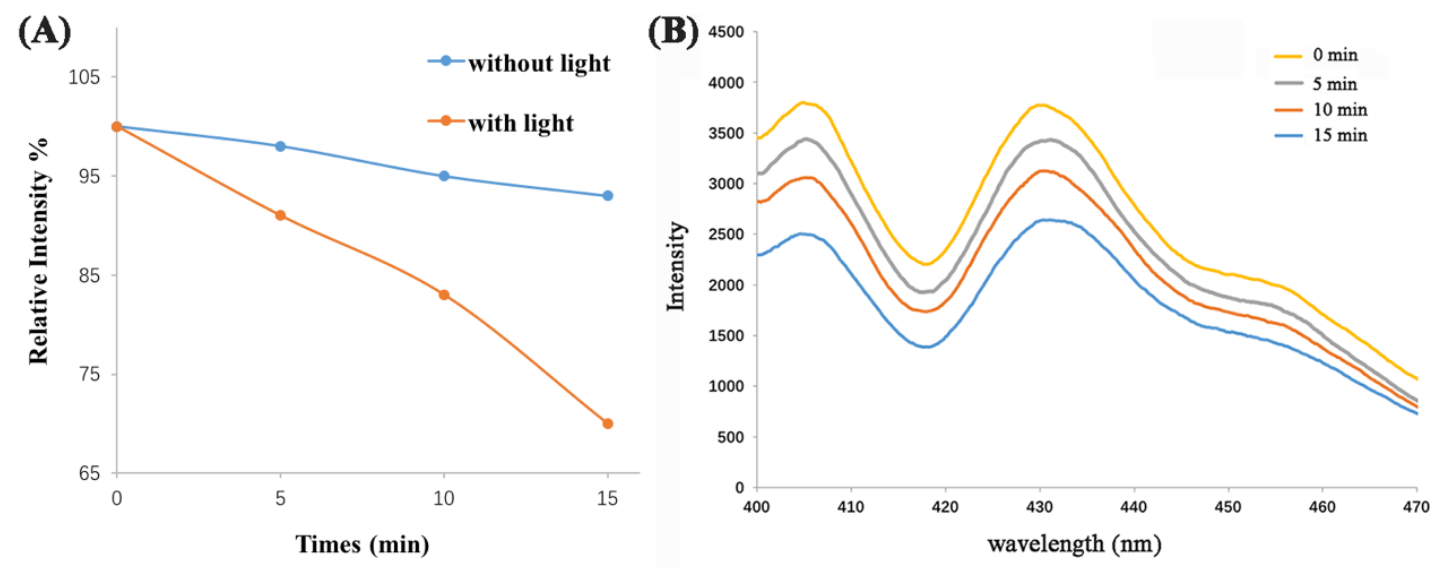

Figure. S10 (A) The relative fluorescence intensity of ABDA based on AuNPs-DOX/PtTPyP@ZIF-8 with or without irradiation at 430 nm. (B) Fluorescence spectrum of ABDA with AuNPs-DOX/PtTPyP@ZIF-8 under irradiation. 